OPEN ACCESS

Edited by:

Takashi Angata,

Academia Sinica, Taiwan

Reviewed by:

Yoshiki Yamaguchi,

Tohoku Medical and Pharmaceutical

University, Japan

Stephan von Gunten,

University of Bern, Switzerland

${ }^{*}$ Correspondence:

Nathalie Juge

Nathalie.Juge@quadram.ac.uk

Specialty section: This article was submitted to

Microbial Immunology,

a section of the journal

Frontiers in Immunology

Received: 19 July 2021 Accepted: 15 September 2021

Published: 01 October 2021

Citation:

Lamprinaki D, Garcia-Vello $P$, Marchetti R, Hellmich C, McCord KA, Bowles KM, Macauley MS, Silipo A, De Castro C, Crocker PR and Juge N (2021) Siglec-7 Mediates Immunomodulation by Colorectal Cancer-Associated Fusobacterium nucleatum ssp. animalis.

Front. Immunol. 12:744184. doi: 10.3389/fimmu.2021.744184

\section{Siglec-7 Mediates}

\section{Immunomodulation by Colorectal Cancer-Associated Fusobacterium nucleatum ssp. animalis}

\author{
Dimitra Lamprinaki ${ }^{1}$, Pilar Garcia-Vello ${ }^{2}$, Roberta Marchetti ${ }^{2}$, Charlotte Hellmich ${ }^{3}$, \\ Kelli A. McCord ${ }^{4}$, Kristian M. Bowles ${ }^{3,5}$, Matthew S. Macauley ${ }^{4}$, Alba Silipo ${ }^{2}$, \\ Cristina De Castro ${ }^{6}$, Paul R. Crocker ${ }^{7}$ and Nathalie Juge ${ }^{1 *}$ \\ ${ }^{1}$ Quadram Institute Bioscience, Norwich Research Park, Norwich, United Kingdom, ${ }^{2}$ Department of Chemical Sciences, \\ University of Naples Federico II, Naples, Italy, ${ }^{3}$ Norfolk and Norwich University Hospitals, NHS Foundation Trust, \\ Norwich, United Kingdom, ${ }^{4}$ Departments of Chemistry, and Medical Microbiology and Immunology, University of Alberta, \\ Edmonton, AB, Canada, ${ }^{5}$ Norwich Medical School, University of East Anglia, Norwich, United Kingdom, ${ }^{6}$ Department of \\ Agricultural Sciences, University of Naples Federico II, Portici, Italy, ${ }^{7}$ Division of Cell Signalling and Immunology, School of \\ Life Sciences, University of Dundee, Dundee, United Kingdom
}

Fusobacterium nucleatum is involved in the development of colorectal cancer (CRC) through innate immune cell modulation. However, the receptors of the interaction between $F$. nucleatum ssp. and immune cells remain largely undetermined. Here, we showed that $F$. nucleatum ssp. animalis interacts with Siglecs (sialic acid-binding immunoglobulin-like lectins) expressed on innate immune cells with highest binding to Siglec-7. Binding to Siglec-7 was also observed using F. nucleatum-derived outer membrane vesicles (OMVs) and lipopolysaccharide (LPS). F. nucleatum and its derived OMVs or LPS induced a pro-inflammatory profile in human monocyte-derived dendritic cells (moDCs) and a tumour associated profile in human monocyte-derived macrophages (moMфs). Siglec-7 silencing in moDCs or CRISPR-cas9 Siglec-7-depletion of U-937 macrophage cells altered $F$. nucleatum induced cytokine but not marker expression. The molecular interaction between Siglec-7 and the LPS O-antigen purified from F. nucleatum ssp. animalis was further characterised by saturation transfer difference (STD) NMR spectroscopy, revealing novel ligands for Siglec-7. Together, these data support a new role for Siglec-7 in mediating immune modulation by F. nucleatum strains and their OMVs through recognition of LPS on the bacterial cell surface. This opens a new dimension in our understanding of how $F$. nucleatum promotes CRC progression through the generation of a pro-inflammatory environment and provides a molecular lead for the development of novel cancer therapeutic approaches targeting F. nucleatum-Siglec7 interaction.

Keywords: Fusobacterium nucleatum, colorectal cancer, Siglec-7, outer membrane vesicle, innate immunity, lipopolysaccharide 


\section{INTRODUCTION}

Colorectal cancer (CRC) is one of the most frequently diagnosed malignancies worldwide, accounting for $10 \%$ of all cancers and for approximately $20 \%$ of all cancer-related deaths in developed countries (1). While CRC incidence commonly appears in ages over 50 years old, recent years have shown an increased incidence in younger adults which may be associated to lifestyle factors $(2,3)$.

Tumours that arise at epithelial barrier surfaces of the body harbour extensive microbiota, and the importance of these microbes in CRC is now widely acknowledged. The enrichment of Fusobacterium spp. in CRC tissues, as revealed by whole genome sequencing, showed that the most abundant species is Fusobacterium nucleatum (4-6). Patients with F. nucleatum associated carcinoma have a shorter survival period (7). In addition, F. nucleatum appears to contribute to the chemoresistance of CRC (8-10). Among F. nucleatum subspecies, $F$. nucleatum ssp. animalis is most predominant in CRC specimens (11).

F. nucleatum potentiates intestinal tumorigenesis mainly by recruitment of tumour infiltrating immune cells, in particular myeloid-derived immune cells such as tumour associated macrophages (TAMs), myeloid-derived suppressor cells (MDSCs), dendritic cells (DCs), tumour associated neutrophils (TANs) (11-13), and inhibits human T-cell response (12, 14, 15), leading to colorectal neoplasia progression. A high abundance of F. nucleatum in CRC tissues is associated with increased nuclear factor kappa B (NFKB) activation and induction of a proinflammatory profile (12). Well-characterised virulence factors of F. nucleatum such as membrane proteins FadA or Fap2 are involved in the binding of $F$. nucleatum to colon cancer cells inducing oncogenic response $(16,17)$. In the epithelium, $F$. nucleatum induces the expression of cell signalling proteins (cytokines), such as tumour necrosis factor (TNF)- $\alpha$ and interleukin (IL)- 8 in addition to epithelial-mesenchymal transition (18). Recently, the structures of O-chain polysaccharides (O-antigens) of the LPS from F. nucleatum strains ATCC 23726 (ssp. nucleatum) (19), ATCC 25586 (ssp. nucleatum) (20), ATCC 10953 (ssp. polymorphum) (21), ATCC 12230 (22), MJR 7757 B (23) and ATCC 51191 (ssp. animalis) (24) have been elucidated, showing strain-specific differences in the trisaccharide repeat unit containing either sialic acid/N-acetylneuraminic acid (Neu5Ac) (21), fusaminic acid (20) or monosaccharides other than nonulosonic acid residues (24). However, the mechanisms underpinning the interaction of F. nucleatum with immune cells remain undefined.

Immune cells express a large variety of glycan-binding receptors or lectins, which sense and respond to changes in the glycan signature of their environment leading to the activation or inhibition of immune processes (25). Siglecs (sialic acid-binding immunoglobulin-like lectins) are a large family of lectins found on innate immune cells and tumourinfiltrating $\mathrm{T}$ cells, which inhibit immune activation after sensing sialic acid-containing glycans $(26,27)$. Individual family members exhibit preferences for sialosides of various linkages to underlying glycan motifs, but many of the physiological ligands, glycoproteins or glycolipids, they interact with remain largely unknown $(28,29)$. The expression of Siglecs on immune cells is cell type dependent (30). Siglecs are transmembrane proteins, which possess an extracellular portion characterized by a $\mathrm{V}$-set immunoglobulin-like domain, containing the carbohydrate recognition domain (CRD), and one or more C2-set immunoglobulin-like domains. The majority of Siglecs possess immunoreceptor tyrosine-based inhibitory motifs (ITIMs) in their intracellular domain (26). Siglec ligands can be presented on the cell on which the Siglec is expressed (cis ligands), or on glycans in the extracellular matrix of other cells (trans ligands) (28). Although the CRDs of most Siglecs have some specificities towards certain sialylated structures, several Siglecs have a broad and overlapping ligand specificity (28). These glyco-immune checkpoints have been proposed as new targets for cancer immunotherapy (31-33). The working hypothesis on the role played by Siglecs in cancer is that immune cells expressing Siglecs are inhibited upon binding to their ligands on cancer cells. Indeed, enzymatic removal of sialic acids from cancer cell surfaces was shown to enhance immune cell-mediated clearance of those cells through loss of Siglec-7 and Siglec-9 binding in cis (34) although the range of physiological ligands of Siglec-7 and Siglec-9 remain to be identified (35). Recently, a genome-wide CRISPR screens revealed the glycoprotein CD43 expressed on leukemia cells as a highly specific ligand for Siglec-7 and blocking the interaction relieved Siglec-7-mediated inhibition of immune killing activity (33). In addition, the tumour immune-suppressive effect of Siglec-7 was recently demonstrated in vivo (36), further supporting the proposed role of Siglec-7 as an immune checkpoint receptor.

Several clinically relevant pathogens have evolved mechanisms of molecular mimicry by displaying sialylated structures on their surface to overcome the cis interactions of Siglecs on the surface of immune cells. For example, Campylobacter jejuni strains can interact with Siglec-7 and sialoadhesin (Siglec-1) via their lipooligosaccharides (37) and to Siglec-10 via a sialic acid-like molecule, pseudaminic acid present in the flagella (38), while Siglec-7 showed sialic acid-independent binding to $\beta$-protein expressed on Group B Streptococcus surface (39). Additionally, human Siglecs have evolved to recognise non-Neu5Ac ligands present on external stimuli such as microbes (40). In this work we hypothesised that Siglecs may be involved in the recognition of $F$. nucleatum strains by immune cells, contributing to the tumorigenesis of these strains in CRC.

\section{MATERIALS AND METHODS}

\section{Materials}

All reagents were purchased from Sigma unless otherwise stated. Recombinant Siglecs, human Siglec-3, Siglec-5, Siglec-7, Siglec-9 and -10 and $\mathrm{CHO}-$ expressing Siglec-7-Fc (CHO-Siglec-7-Fc) cell line were a kind gift from Prof. Paul Crocker (University of Dundee). Recombinant Siglec-7-Fc was also obtained commercially (R\&D Systems).

\section{Bacteria Growth, Preparation and Quantification}

F. nucleatum ssp. animalis ATCC 51191 isolated from clinical samples was obtained from ATCC in partnership with LGC 
standards ltd. F. nucleatum was cultured in tryptic soy broth media (Becton Dickinson) supplemented with $5 \mu \mathrm{g} / \mathrm{ml}$ hemin (Sigma) and $1 \mu \mathrm{g} / \mathrm{ml}$ menadione (Sigma). For binding and human cell co-culture experiments, bacteria were centrifuged at $15,000 \mathrm{x} \mathrm{g}$ for $5 \mathrm{~min}$, and the cells were fixed with $4 \%$ paraformaldehyde (PFA) (Electron Microscopy Sciences/CN Technical Services ltd) for $45 \mathrm{~min}$ at room temperature (RT), in the dark, followed by two washes in Dulbecco's Phosphatebuffered Saline (DPBS) (Lonza).

For bacteria de-sialylation, $10^{7}$ cells were treated with $20 \mathrm{U}$ of sialidase $\alpha 2-3,6,8,9$ neuraminidase A (NEB) in $1 X$ GlycoBuffer I (NEB) or control treated in $1 \mathrm{X}$ GlycoBuffer I alone, overnight $(\mathrm{o} / \mathrm{n})$ at $37^{\circ} \mathrm{C}$.

Bacteria were quantified by spectroscopy with $\mathrm{OD}_{600 \mathrm{~nm}}$ of 1 corresponding to $10^{9}$ cells $/ \mathrm{ml}$ or by imaging flow cytometry (Amnis ImageStream ${ }^{\mathrm{x}} \mathrm{Mk}$ II) (as described below).

\section{LPS Extraction}

F. nucleatum ATCC 51191 bacterial cells were harvested by centrifugation, lyophilised, and extracted by the hot phenol/water method, as previously described (41). Each phase was dialysed against distilled water to remove the phenol, freeze-dried, and analysed by $12 \%$ sodium dodecyl sulphate polyacrylamide gel electrophoresis (SDS-PAGE). After the water/phenol extraction, F. nucleatum LPS extracted from was detected in the water phase by silver nitrate staining (42). The phases containing LPS were further purified by enzymatic digestion (DNAse, RNAse and proteinase K) as previously described (41), followed by centrifugation at 6,000 $\mathrm{rpm}$ for $30 \mathrm{~min}$ at $4^{\circ} \mathrm{C}$ and ultracentrifugation at $30,000 \mathrm{rpm}$ for $4 \mathrm{~h}$ at $4^{\circ} \mathrm{C}$. To separate the $\mathrm{O}$-antigen (OPS) and lipid A domains, LPS were mild acid hydrolysed by acetic acid $1 \%\left(100^{\circ} \mathrm{C}, 2-3 \mathrm{~h}\right)$. The OPS domain of the strains containing ulosonic residues was further partial depolymerised. The solution was centrifuged and the supernatants were freeze-dried and further purified by gel filtration chromatography.

\section{F. nucleatum OMV Purification and Characterisation}

F. nucleatum derived OMVs were collected from the cell culture supernatant, as described previously by Liu et al. (43) with some modifications. Briefly, F. nucleatum ssp. cells were cultured until reaching $\mathrm{OD}_{600 \mathrm{~nm}}$ of 0.7-1.2. Cells were centrifuged at $8,500 \mathrm{x} \mathrm{g}$ for $15 \mathrm{~min}$ at $4^{\circ} \mathrm{C}$. The supernatant was collected, and vacuum filtered using $0.22 \mu \mathrm{m}$ membrane. The filtered supernatant was concentrated by spin-filtration using 100,000 molecular weight cut-off filter unit (Sartorius). OMVs were recovered from the filter using sterile DPBS and further purified by density gradient ultra-centrifugation. For the gradient, Optiprep media $(60 \% \mathrm{w} / \mathrm{v}$, Sigma) was diluted in $0.85 \% \mathrm{w} / \mathrm{v} \mathrm{NaCl}$ and $10 \mathrm{mM}$ tricine- $\mathrm{NaOH}$ pH 7.4 solution to make $35 \%, 30 \%, 25 \%$ and $20 \%$ density solutions. The OMVs were mixed with $40 \%$ Optiprep solution and placed at the bottom of a $13.2 \mathrm{ml}$ Ultra-clear tube (Beckman Coulter) and Optiprep $(2 \mathrm{ml})$ was added subsequently by density-decreasing order. The preparation was ultracentrifuged at $135,000 \mathrm{x}$ g for $16 \mathrm{~h}$ at $4^{\circ} \mathrm{C}$ with minimum acceleration and deceleration using a SW41 Ti rotor (Beckman Coulter). From the top to the bottom, $1 \mathrm{ml}$ fractions were collected and analysed by SDS-PAGE in $4-15 \%$ Mini-PROTEAN ${ }^{\circledR}$ TGX $^{\mathrm{TM}}$ Precast Protein
Gel (BIO-RAD). The OMV-containing fractions were diluted with sterile DPBS and ultracentrifuged at 200,500 $\mathrm{x}$ g for $2 \mathrm{~h}$ at $4^{\circ} \mathrm{C}$ using a Type 45 Ti rotor (Beckman Coulter). OMVs were resuspended in sterile DPBS and then filtered using a $0.22 \mu \mathrm{m}$ membrane.

Purified OMVs were quantified and measured for their particle size using a NanoSight LM12 (Malvern Panalytical). Briefly, the samples were diluted 100 times in $1 \mathrm{ml}$ DPBS and loaded onto the instrument's chamber by a syringe and the sample were slowly released. The considered particle size of each OMV sample were the mean of triplicates. Instrument settings used: camera shutter 1035, camera gain 680, capture duration $60 \mathrm{sec}$.

\section{Semi-Quantitative Analysis of LPS in OMVs by Gas Chromatography-Mass Spectrometry (GC-MS)}

The content of LPS in F. nucleatum-derived OMVs was evaluated by analysing the fatty acids content. Based on the chemical structure of the lipid A component of F. nucleatum LPS in the bacteria (44), C14:0 (or myristic acid) was considered as the reporter group for LPS, while C16:0 (palmitic acid) and C18:0 (stearic acid) were considered the reporters for the phospholipids.

F. nucleatum-derived OMVs (1 mg) were treated with $\mathrm{HCl} /$ $\mathrm{MeOH}\left(1 \mathrm{ml}, 1.25 \mathrm{M}, 80^{\circ} \mathrm{C}, 16 \mathrm{~h}\right)$ and lipids, derivatised as methylesters, were extracted with hexane (41). This analysis estimated the amount of each fatty acid (C14:0, C16:0 and C18:0) by correcting the areas of the corresponding peaks with a response factor, made by using an array of standard solutions and by setting C16:0 as internal standard. Areas were correlated by a linear regression. The methanol layer after extraction with hexane, was used to countercheck the data from lipid analysis, by verifying the presence of 3-deoxy-2-keto-D-manno-octulosonic acid and L-glycero-D-manno-heptose, both markers of the LPS molecules. Identification of the fatty acids or the monosaccharide constituents, was performed by comparing the retention time and the fragmentation pattern of each peak to a relevant standard.

All chemical derivatives were analysed by using a Gas Chromatography-Mass Spectrometry (GC-MS) Agilent Technologies 7820A (Santa Clara, CA, USA) equipped with a mass selective detector $5977 \mathrm{~B}$ and a HP-5ms capillary column Agilent, Italy ( $30 \mathrm{~m} \times 0.25 \mathrm{~mm}$ i.d., $0.25 \mu \mathrm{m}$ as film thickness, flow rate $1 \mathrm{ml} / \mathrm{min}$, He as carrier gas). Electron impact mass spectra were recorded with ionisation energy of $70 \mathrm{eV}$ and an ionising current of $0.2 \mathrm{~mA}$. The temperature program used was: $150^{\circ} \mathrm{C}$ for $5 \mathrm{~min}, 150$ up to $300^{\circ} \mathrm{C}$ at $10^{\circ} \mathrm{C} / \mathrm{min}, 300^{\circ} \mathrm{C}$ for $12 \mathrm{~min}$.

\section{Expression and Purification of Recombinant Siglec-Fc Proteins}

CHO-Siglec-7-Fc cells were cultured in Glasgow Modified Essential Medium (GMEM) without L-glutamine media (Sigma) supplemented with $10 \%$ fetal bovine serum (FBS) (Thermo Scientific Gibco), $100 \mathrm{U} / \mathrm{ml}$ penicillin and $100 \mu \mathrm{g} / \mathrm{ml}$ streptomycin (Lonza) and 50X GS supplements (Sigma).

Adherent CHO-Siglec-7-Fc cells (80-90\% confluence) were washed twice with Dulbecco's phosphate-buffered saline (DPBS) (Thermo Scientific Gibco) and protein expression was induced by culturing the cells with GMEM without L-glutamine media (Sigma) supplemented with 200X FetalClone II (Thermo Fisher 
Scientific), $100 \mathrm{U} / \mathrm{ml}$ penicillin and $100 \mu \mathrm{g} / \mathrm{ml}$ streptomycin (Lonza), 50X GS supplements (Sigma) and $100 \mu \mathrm{g} / \mathrm{ml} \mathrm{MSX}$ (Sigma). After 4 days, the supernatant was collected for Siglec7-Fc purification.

Siglec-7-Fc purification was carried out using gravity-flow column (BIO-RAD) packed with protein A-Sepharose (Sigma) washed with DPBS. The harvested CHO supernatant was added to the column and the column washed with DPBS. To elute Siglec-7-Fc, a solution of $0.1 \mathrm{M}$ glycine, $\mathrm{pH} 3$ was added to the column and fractions $(0.5 \mathrm{ml})$ were collected in $1 \mathrm{M}$ Tris, $\mathrm{pH} 8$ (for neutralisation). The protein concentration in fractions was quantified by Nanodrop (Thermo Fisher Scientific).

\section{Binding Assays}

For the flow cytometry binding assays between F. nucleatum ssp. animalis ATCC 51191 and recombinant Siglecs (Siglec-3, Siglec5 , Siglec-7, Siglec- 9 and -10$)$, bacteria $\left(10^{7}\right.$ cells) were incubated with the pre-complex of recombinant Siglec-Fc $(4 \mu \mathrm{g} / \mathrm{ml})$ and mouse $\alpha$-Fc-PE Ab $(1 \mu \mathrm{g} / \mathrm{ml})$ (R\&D Systems) in DPBS for $1 \mathrm{~h}$ at $37^{\circ} \mathrm{C}$. Following centrifugation at $14,000 \mathrm{x}$ g for $4 \mathrm{~min}$, bacterial cells were washed with DPBS and analysed by Fortessa (BD Biosciences). For the inhibition assays, Siglec-7-Fc and a-Fcphycoerythrin (PE) Ab pre-complex was first incubated with disialoganglioside with three glycosyl groups GD3 (Sigma) at 50 $\mu \mathrm{g} / \mathrm{ml}$ for $30 \mathrm{~min}$ at $4^{\circ} \mathrm{C}$. For the flow cytometry binding assays between F. nucleatum ssp. and human cells, U-937 (WT or Siglec- $7^{-/-}$) cells were first stained with $10,000 \mathrm{X}$ cell trace violet (CTV) (Thermo Fisher Scientific) for $15 \mathrm{~min}$ at RT and $F$. nucleatum ssp. were stained with $10 \mu \mathrm{g} / \mathrm{ml}$ of fluorescein isothiocyanate (FITC) (Sigma). Following two washes with DPBS, U-937-CTV ( $5 \times 10^{5}$ cells) were incubated with F. nucleatum-FITC $\left(5 \times 10^{6}\right.$ cells) for $1 \mathrm{~h}$ at $4^{\circ} \mathrm{C}$. Cells were washed with FACS buffer (HBSS containing $0.01 \%$ bovine serum albumin (BSA), and $2 \mathrm{mM}$ EDTA), centrifuged at $510 \mathrm{xg}$ for $3 \mathrm{~min}$ and analysed using Fortessa (Threshold of FSC parameter set to 1000). Flow cytometry data were processed in FlowJo (TreeStar) software.

For the ELISA-based binding assays, bacteria $\left(10^{7}\right.$ cells) or bacteria-derivatives $\left(10 \mu \mathrm{g} / \mathrm{ml}\right.$ LPS or $10^{8} \mathrm{OMV}$ particles $)$ in DPBS solution were coated in a 96 -well plate, o/n at $4^{\circ} \mathrm{C}$. Following a washing step with $0.05 \%$ tween in PBS (washing buffer) the plate was incubated with $1 \%$ BSA for $1 \mathrm{~h}$ at RT. Followed by 3 times washing the plates were incubated with precomplexed Siglec-Fc and $\alpha$-Fc-HRP for $2 \mathrm{~h}$ at RT. Briefly, SiglecFc protein $(4 \mu \mathrm{g} / \mathrm{ml})$ was incubated with $50,000 \mathrm{X} \alpha$-human-FcHRP (Abcam) for $1 \mathrm{~h}$ at RT. Following 3 washes with washing buffer, the plate was incubated with $3,3^{\prime}, 5,5^{\prime}$ tetramethylbenzidine (TMB) (Biolegend) until colour development. Colour development was stopped by the addition of $2 \mathrm{~N} \mathrm{H}_{2} \mathrm{SO}_{4}$ and the absorbance was measured at $450 \mathrm{~nm}$ with reference at $570 \mathrm{~nm}$. Data were analysed in GraphPad Prism 6.

\section{STD NMR Analysis}

Spectra were acquired on a Bruker $600 \mathrm{MHz}$ AVANCE NEO equipped with a cryo probe and analysed using the TOPSPIN 4.1.0 software. The partial depolymerised OPS derived from F. nucleatum ssp. LPS were prepared in deuterated PBS buffer (20 mM PBS, $\mathrm{NaCl} 150 \mathrm{mM}, \mathrm{pH}=7.4$ ), using protein-ligand ratios varying from 1: 20 to 1: 80 with $15 \mu \mathrm{M}$ of Siglec-7-Fc protein. STD NMR experiments were acquired at $298 \mathrm{~K}$ with $32 \mathrm{k}$ data points and zero-filled up to $64 \mathrm{k}$ data points prior to processing. The Siglec-7-Fc resonances were saturated applying 40 Gauss pulses with a length of $50 \mathrm{~ms}$, setting the on-resonance pulse at aromatic region (7.5/6-5 ppm) and the off-resonance pulse frequency at $100 \mathrm{ppm}$. Under these experimental conditions, very low residual signals were observed in some of the STD NMR spectra for the ligands in the free state which were taken into account during data processing. To suppress the water signal, an excitation sculpting with gradient pulses (esgp) was applied and to reduce the NMR signals of Siglec-7-Fc, a spin-lock filter (20 ms) was used.

\section{Culture of Human Primary Immune Cells and U-937 Monocytic Cell Line}

Human peripheral blood was obtained from haemochromatosis patients undergoing a therapeutic venesection at the Norfolk and Norwich University Hospital (Norwich, UK). Blood collection in this study was approved by the Faculty of Medicine and Health Sciences Research Ethics Committee REC reference number 2013/2014 -14HT (University of East Anglia).

For monocyte-derived dendritic cell (moDC) and macrophage $(\mathrm{moM} \phi)$ generation, peripheral blood mononuclear cells (PBMCs) were isolated from whole blood following centrifugation using Ficoll-Paque gradient media (GE Healthcare). Monocytes (CD14+ cells) were isolated from PBMCs using CD14 positive selection microbeads (StemCell technologies) according to the manufacturer's instructions. Freshly isolated CD14+ monocytes $\left(10^{6}\right.$ cells $\left./ \mathrm{ml}\right)$ were cultured in Mercedes medium (RPMI 1640 medium (Lonza) supplemented with $25 \mathrm{mM}$ HEPES, 10\% FBS (Thermo Scientific Gibco), $55 \mu \mathrm{M}$ 2-mercaptoethanol, $100 \mathrm{U} / \mathrm{ml}$ penicillin and $100 \mu \mathrm{g} / \mathrm{ml}$ streptomycin (Lonza), $2 \mathrm{mM}$ glutamine (Lonza), $1 \mathrm{mM}$ non-essential amino acids (Lonza) and $1 \mathrm{mM}$ sodium pyruvate (Lonza), were incubated with granulocytemacrophage colony-stimulating factor (GM-CSF) and IL-4 (PeproTech) (25 ng/ml) for differentiation of monocytes to moDCs or with macrophage colony-stimulating factor (M-CSF) (PeproTech) $(25 \mathrm{ng} / \mathrm{ml})$ for differentiation of monocytes to moM $\phi$ s. The cells were incubated for 7 days at $37^{\circ} \mathrm{C}$, with addition of the above cytokines on day 3, as previously described (45).

For U-937 differentiation, U-937 ( $5 \times 10^{5}$ cells $\left./ \mathrm{ml}\right)$ cultured in RPMI 1640 medium (Lonza) supplemented with 25 mM HEPES, 10\% FBS (Thermo Scientific Gibco), $55 \mu \mathrm{M}$ 2-mercaptoethanol, $100 \mathrm{U} / \mathrm{ml}$ penicillin and $100 \mu \mathrm{g} / \mathrm{ml}$ streptomycin (Lonza), $2 \mathrm{mM}$ glutamine (Lonza) and $1 \mathrm{mM}$ sodium pyruvate (Lonza) were differentiated with $100 \mathrm{ng} / \mathrm{ml}$ phorbol 12-myristate 13-acetate (PMA) (Sigma) for $28 \mathrm{~h}$ at $37^{\circ} \mathrm{C}$, as previously described (46). Adherent cells were detached by PBS-EDTA (Lonza) and scraping and collected for functional assays.

\section{Generation of Siglec-7/- U-937 Cells by CRISPR-Cas9}

CRISPR RNA (crRNA) was designed to target human Siglec-7 (CATGCCCTCTTGCACGGTCA, IDT) in U-937 cells (ATCC ${ }^{\circledR}$ CRL-1593.2 $\left.{ }^{\mathrm{TM}}\right)$. guide RNA (gRNA) $(1 \mu \mathrm{M}$ crRNA, $1 \mu \mathrm{M}$ ATTO-550 labeled tracrRNA (IDT)) was boiled at $95^{\circ} \mathrm{C}$ for 
$5 \mathrm{~min}$. A solution of $20 \mathrm{pmol}$ gRNA, $20 \mathrm{pmol}$ Cas9 nuclease (IDT), $8 \mu$ Cas9 PLUS reagent (IDT), $16 \mu \mathrm{l}$ CRISPRMAX reagent (Thermo Fisher) in $600 \mu \mathrm{l}$ of Opti-MEM medium (Gibco) was prepared. 750,000 U-937 cells were washed with Opti-MEM medium (Gibco) and centrifuged at $300 \mathrm{x}$ g for $5 \mathrm{~min}$. The cell pellet was resuspended in the prepared solution and incubated at $37^{\circ} \mathrm{C}, 5 \% \mathrm{CO}_{2}$. After a $24 \mathrm{~h}$ incubation, cells were centrifuged at $300 \mathrm{x} \mathrm{g}$ for $5 \mathrm{~min}$, then resuspended in $400 \mu$ flow buffer (HBSS, $1 \%$ FBS, $500 \mu \mathrm{M}$ EDTA). The top 5\% ATTO-550 positive cells were sorted on a BD FACSMelody ${ }^{\mathrm{TM}}$ Cell Sorter into four 96-well flat-bottom plates containing media at one cell per well. Approximately 2 weeks later, colonies were screened for Siglec-7 expression by flow cytometry using PE-conjugated Siglec-7 at 1:100 dilution (BioLegend) and Siglec- $7^{-/-}$clones were collected.

\section{Siglec-7 RNA Silencing of Primary Immune Cells}

moDCs were transfected with two pre-designed small interfering RNA (siRNA) Silencer Select SIGLEC7 probes (ID\# s25729 and s25730) or with the scramble siRNA (Invitrogen) with reverse transfection, as described previously (47). Briefly, $3 \times 10^{5}$ moDC or moM $\phi$ cells were incubated with the complex of two probes to a final $200 \mathrm{nM}$ concentration or with the negative control (scramble) and 1\% HiPerFect transfectant (Qiagen) in warm RPMI 1640 (non-supplemented) media in a 24-well plate for three days.

\section{Cytokine and Cell Surface Marker Analysis}

moDCs or moM $\phi$ s or U-937-PMA $\left(10^{5}\right.$ cells $)$ were cultured in 96-well plates in the Mercedes medium as described above and stimulated with PFA-fixed F. nucleatum ssp. at multiplicity of infection (MOI) of 50 or 5, F. nucleatum ssp.-derived LPS at 10 or 1 $\mu \mathrm{g} / \mathrm{ml}$, the E. coli O111:B4 control at $1 \mu \mathrm{g} / \mathrm{ml}$, or OMVs at $5 \times 10^{7}$ particles $/ \mathrm{ml}$ for $18 \mathrm{~h}$ at $37^{\circ} \mathrm{C}$. The cells were centrifuged at $510 \mathrm{xg}$ for $3 \mathrm{~min}$ and the supernatant and pellet collected for analysis.

For cytokine analysis, human TNF $\alpha$, IL-10, IL-8 production in the supernatant was quantified by ELISA (BioLegend) according to the manufacturer's instructions.

For cell surface marker analysis, moDC or moM $\phi$ pellets were first incubated with human Fc block (BioLegend) and then incubated with antibodies for $30 \mathrm{~min}$ at $4^{\circ} \mathrm{C}$ as follows: programmed death-ligand 1 (PD-L1)-PE at 1:50 dilution or CD80-PE at 1:100 dilution, CD86Alexa488 at 1:200 dilution, isotype controls mouse IgG1-PE, $\kappa$ at 1:100 (BioLegend) at 1:25 dilution (BioLegend) and with propidium iodide (PI) or 4'6-diamidino-2-phenylindole (DAPI) at 0.1 or $1 \mu \mathrm{g} /$ $\mathrm{ml}$ for dead cell staining, respectively. The cells were then washed with DPBS supplemented with 1\% BSA (FACS buffer) analysed by flow cytometry using Fortessa.

\section{Imaging Flow Cytometry}

For counting bacteria, 10,000 events were collected and processed using the IDEAS (Amnis) software. Bacteria density "objects/ml" were selected in the bright field channel (M04) of the Aspect Ratio_M04 versus Area_M04 dot plot.

For internalisation assays, human cells (monocyte-derived or U-937-PMA) at $5 \times 10^{6}$ cells/ml were incubated with $5 \times 10^{7}$ FITC-stained F. nucleatum ssp. for 4 h. Cells were washed with
FACS buffer and analysed by ImageStreamx Mk II (Amnis). Using the INSPIRE software, a total of 5,000 FITC stained cells were collected. The percentage of internalised bacteria were determined using the internalisation wizard with erode mask function at 7 number of pixels.

\section{Statistical Analyses}

One-way ANOVA followed by Tukey's test were used for multiple comparisons, t-test or two-way ANOVA were used for two-group comparisons, on Prism software (GraphPad). P < 0.05 was considered as statistically significant. ${ }^{*} \mathrm{p}<0.05,{ }^{* *} \mathrm{p}<$ $0.01,{ }^{* *} \mathrm{p}<0.001,{ }^{* * * *} \mathrm{p}<0.0001$, n.s., not statistically difference.

\section{RESULTS}

\section{F. nucleatum ssp. animalis Binding to Siglecs Revealed Specific Binding to Siglec-7}

The binding of F. nucleatum ATCC 51191 was first tested against a range of human recombinant CD33-related Siglec-Fc proteins including Siglec-3, Siglec-5, Siglec-7, Siglec- 9 and -10 by flow cytometry. A clear shift in fluorescence was observed in the presence of Siglec-7 with approx. $90 \%$ of F. nucleatum population bound to Siglec-7, while $60 \%$ and $30 \%$ of F. nucleatum population bound to Siglec-5 and Siglec-3, respectively, and only $17 \%$ of the population bound to Siglec-9 and -10 (Figure 1A). To determine if the binding to Siglec-7 was glycan-mediated, inhibition binding assays were carried out in the presence of ganglioside GD3, a known Siglec-7 ligand (48). A significant decrease in Siglec-7 binding to $F$. nucleatum ATCC 51191 was observed in the presence of GD3, showing an approx. 92\% reduction of the bacterial cell population bound to Siglec-7 (Figure 1B). This result suggests that Siglec-7 Vset domain is implicated in the binding between Siglec-7 and F. nucleatum ssp. To investigate whether the binding of F. nucleatum ATCC 51191 to Siglec-7 was mediated by sialic acid exposed on the bacterial cell surface, the bacterial cells were treated with neuraminidase $A$, a sialidase with broad specificity to $(\alpha 2-3,6,8,9)$ sialylated linkages, cleaving linear and branched non-reducing terminal sialic acid residues from glycoconjugates. The sialidase pre-treatment only led to a small reduction in the binding of $F$. nucleatum to Siglec-7 as shown by flow cytometry (Figure S1), in agreement with the absence of sialic acid in F. nucleatum ATCC 51191 LPS (24). Next, we conducted binding assays between the monocytic cell line U-937 (wild type (WT) or Siglec- $7^{-/}$) and F. nucleatum ATCC 51191 by flow cytometry. Our results showed a reduction of F. nucleatum associated with U-937-Siglec- $7^{-/-}$cells as compared to WT cells (Figure 1C), supporting an interaction between F. nucleatum ssp. and Siglec-7 when expressed on the cell surface although other receptors may be involved in the interaction between F. nucleatum ATCC 51191 and U-937 cells.

\section{F. nucleatum Derived LPS or OMVs Bind to Siglec-7}

To determine the role of LPS in the binding of F. nucleatum ATCC 51191 to Siglec-7, LPS was extracted from F. nucleatum 
A

Fn ATCC 51191

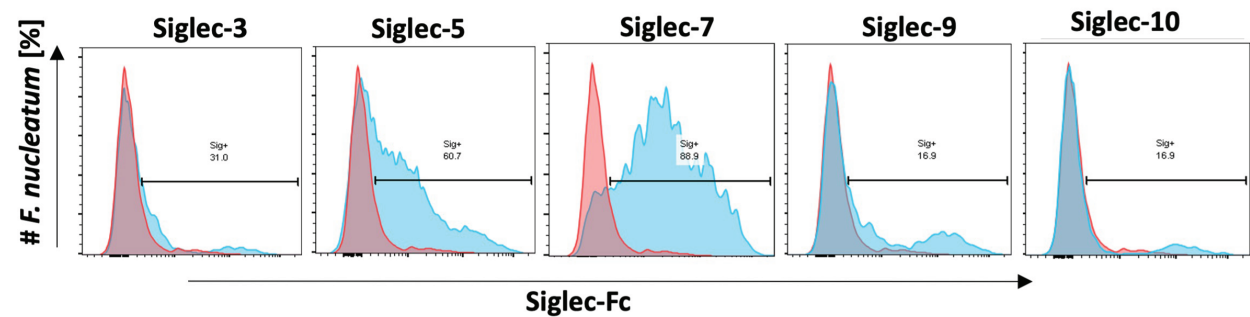

B

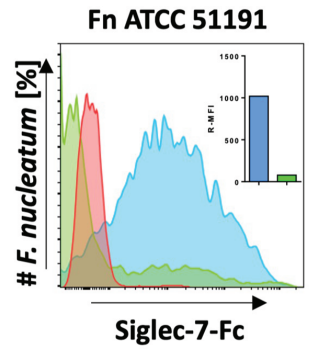

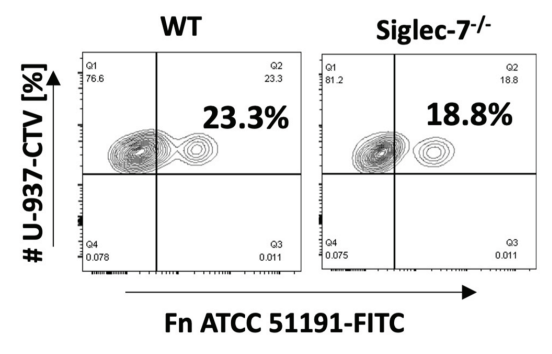

FIGURE 1 | Binding of $F$. nucleatum ATCC 51191 to Siglecs using flow cytometry. (A) Binding of $F$. nucleatum to recombinant Siglec-Fc proteins (in blue). (B) Binding of $F$. nucleatum to Siglec-7 in the presence of GD3 inhibitor (in green) or untreated cells (in blue). (C) Binding of $F$. nucleatum to WT or Siglec- $7^{-1-}$ U-937 cells. Bacteria incubated with $\alpha$-Fc-PE Ab only was used as a control (in red). Fn, F. nucleatum.

ATCC 51191 by the hot phenol/water method (41) and further purified by enzymatic digestion (41). The SDS-PAGE of the extracted F. nucleatum-derived LPS showed the typical LPS ladder-like pattern and lower average molecular weight distribution when compared to E. coli O127:B8 (Figure S2). Using an ELISA-type assay, we showed that the whole $F$. nucleatum cells and the derived LPS bound to Siglec-7-Fc (Figures 2A, B) while no binding of $F$. nucleatum-derived LPS was observed against Siglec-9-Fc used as a control (Figure 2B). To map the relevant positions of $F$. nucleatum LPS involved in the interaction with Siglec-7 and gain a first evaluation of the ligand epitopes, the partially depolymerised O-antigen chain (OPS) isolated from F. nucleatum ATCC 51191 was analysed by STD NMR (49) (Figure 2D). Interestingly, STD enhancements, together with changes in the relative intensity of STD signals with respect to the reference spectrum, were detected, clearly indicating that $F$. nucleatum ATCC 51191 OPS structure was recognised by and interacted with Siglec-7-Fc. Despite the significant overlapping of ligand resonances which impaired a detailed analysis of the protons involved in the recognition and binding process, the fingerprint of STD NMR spectrum allowed to identify the ligand regions in close contact with Siglec-7-Fc. F. nucleatum ATCC 51191 OPS contains a linear trisaccharide made up of glucosaminuronic (GlcNAcA and GlcNAc3NAlaA) and fucosamine $($ FucNAc4N) residues, $[\rightarrow 4)-\beta$ D-GlcpNAcA- $(1 \rightarrow 4)-\beta$-D-GlcpNAc3NAlaA- $(1 \rightarrow 3)-\alpha-D$ FucpNAc4NR- $(1 \rightarrow]$, with the N-4 of the fucosamine partly acetylated $(60 \%)$. The analysis of signals in isolated regions of the spectrum, i.e., in the range between $0.8-1.5 \mathrm{ppm}$, demonstrated the contribution to the interaction from glucosaminuronic (GlcNAcA and GlcNAc3NAlaA) and fucosamine (FucNAc4N) residues. Therefore, STD NMR analysis confirmed binding of Siglec-7 to F. nucleatum ATCC 51191 OPS, even though it lacks nonulosonic acid residues (Figure 2D).

Next, we purified outer-membrane vesicles (OMVs) produced by $F$. nucleatum ATCC 51191 by density gradient ultracentrifugation, resulting in pure and spherical particles with a diameter range from 30 to $250 \mathrm{~nm}$ (Figure S3A). We showed by GC-MS that LPS is a main constituent (approximately in 60$70 \% \mathrm{~mol} / \mathrm{mol}$ ) of F. nucleatum-derived OMVs (Figure S3B). We then tested the ability of F. nucleatum-derived OMVs to bind to Siglec-7 (Figure 2C). F. nucleatum-derived OMVs from ATCC 51191 bound to Siglec-7 at levels comparable to LPS under the conditions tested (Figure 2C).

Together these data identified F. nucleatum LPS present on whole cells or OMVs as a new ligand to Siglec-7-Fc.

\section{F. nucleatum Modulates Immune Response in a Cell Subset Specific Manner}

To investigate the impact of $F$. nucleatum ssp. on the host immune response, myeloid cells, moDCs and moM $\phi$ s, were generated from human blood, and stimulated with F. nucleatum ATCC 51191 or with F. nucleatum ATCC 51191-derived LPS and OMVs.

F. nucleatum bacterial cells were shown to associate with the cell surface of moDCs or moM $\phi$ s as determined by imaging flow cytometry (Figure S4). Stimulation of moDCs with F. nucleatum at MOI 5 resulted in a marked increase in cytokine production of TNFo, IL-8 ( $<$ 0.0001) (Figure 3A) and an induction of CD86 


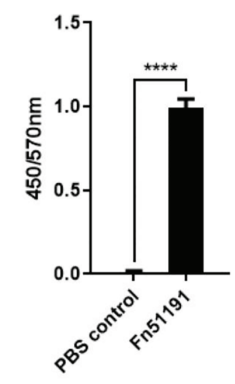

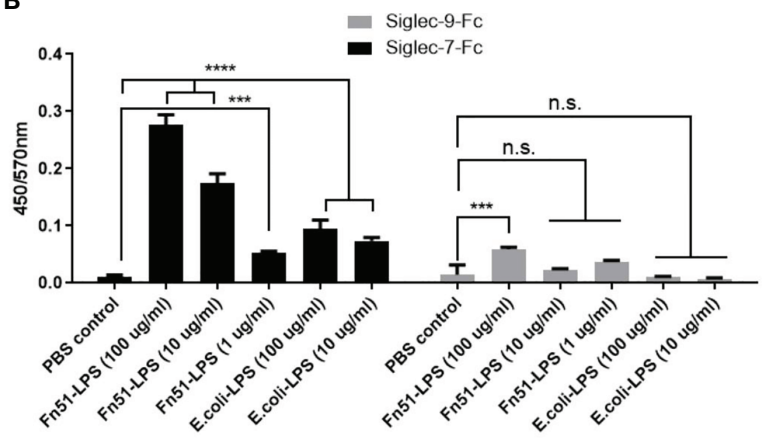

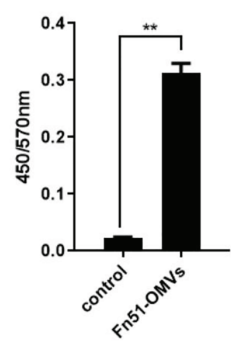

D

\section{F. nucleatum ATCC 51191}
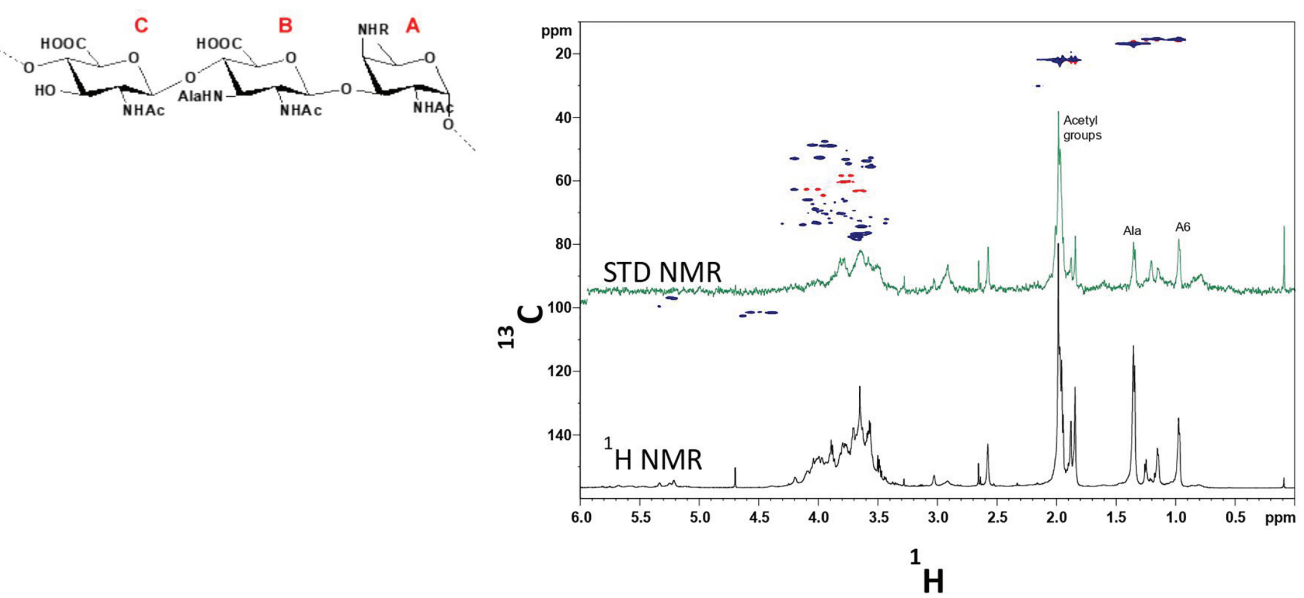

FIGURE 2 | Binding of F. nucleatum ATCC 51191 cells, LPS or OMVs to Siglec-7-Fc. Immobilised F. nucleatum 51191 cells were tested for binding to (A) Siglec-7Fc and the extracted LPS to (B) Siglec-7-Fc or Siglec-9-Fc by ELISA. (C) Immobilised OMVs extracted from F. nucleatum 51191 were tested for binding to Siglec-7Fc by ELISA. PBS was used as control. Data shown are the mean of duplicates \pm SD derived from one representative experiment reproduced in three independent experiments. Fn, F. nucleatum. (D) STD NMR analysis of the binding between Siglec-7 and partially depolymerised OPS from F. nucleatum ATCC 51191. The panel shows the superimposition of the reference ${ }^{1} \mathrm{H}$ NMR spectrum (in black) and STD NMR spectrum (in green), the ${ }^{1} \mathrm{H}-{ }^{13} \mathrm{C}$ HSQC spectrum (blue/red) and the chemical structure of $F$. nucleatum OPS repeating units. Statistical analyses were performed by t-test (for panels 2Aand 2C) or one-way ANOVA followed by Tukey's test. $\mathrm{P}<0.05$ was considered as statistically significant. ${ }^{* \star} \mathrm{p}<0.01,{ }^{* * \star} \mathrm{p}<0.001,{ }^{* \star * *} \mathrm{p}<0.0001$, n.s., not statistically difference.

and PD-L1 as compared to the unstimulated control (Figure 3B). A different profile was observed with moM $\phi$ s, where stimulation with $F$. nucleatum led to a significant induction of IL-10 and IL-8 production ( $\mathrm{p}<0.0001$ ), low levels (n.s.) of TNF $\alpha$ production (Figure 3A) and to the induction of PD-L1 and downregulation of CD86 cell surface markers as compared to the unstimulated control (Figure 3B). The results were dose-dependent, with a marked increase in cytokine production when cells were stimulated at MOI 50 as compared to MOI 5 (Figure S5A). This acquired moM $\phi$ phenotype was also observed using the macrophage like cell line U-937 after F. nucleatum ATCC 51191 stimulation, leading to high IL-10 and low TNF $\alpha$ levels (Figure S5B). Consistent with these results, we showed, using imaging flow cytometry, that both moDCs and moMфs were able to internalise F. nucleatum (Figure 3C), with
moDCs showing approx. $10 \%$ less internalisation as compared to moMфs (Figure 3C).

Next, we stimulated moDCs or moMфs with $F$. nucleatum ATCC 51191-derived LPS or OMVs. In moDCs, treatment with OMVs or with LPS at $10 \mu \mathrm{g} / \mathrm{ml}$ but not $1 \mu \mathrm{g} / \mathrm{ml}$ induced TNF $\alpha$ production (Figures 4A, B). In moM $\phi s$, stimulation OMVs or LPS (at both 10 or $1 \mu \mathrm{g} / \mathrm{ml}$ ) showed significant induction of IL-10 at levels comparable to the whole bacteria (Figures 4A, B). When moM $\phi$ s or moDCs were treated with LPS or OMVs, there was an upregulation of the CD80 cell surface marker expression as compared to the unstimulated control, as showed with the whole bacteria. In moDCs, LPS stimulation led to an induction of CD86 expression (Figure 4C), as also observed with the whole bacteria, while stimulation with OMVs showed a reduction of CD86 expression compared to the unstimulated control 
A
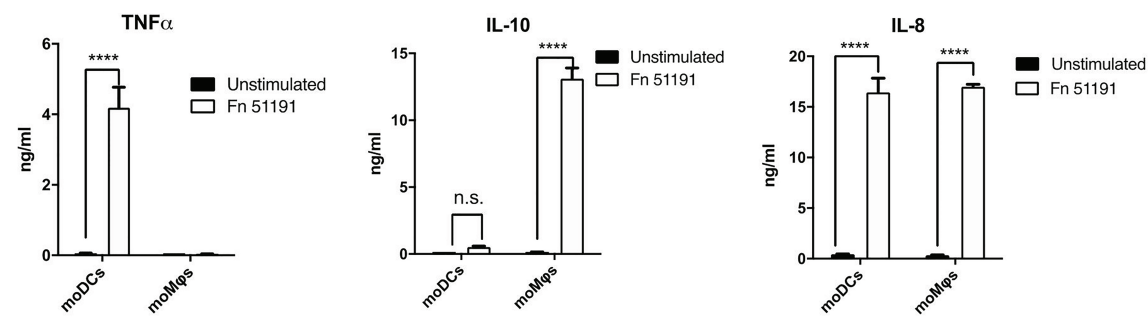

B
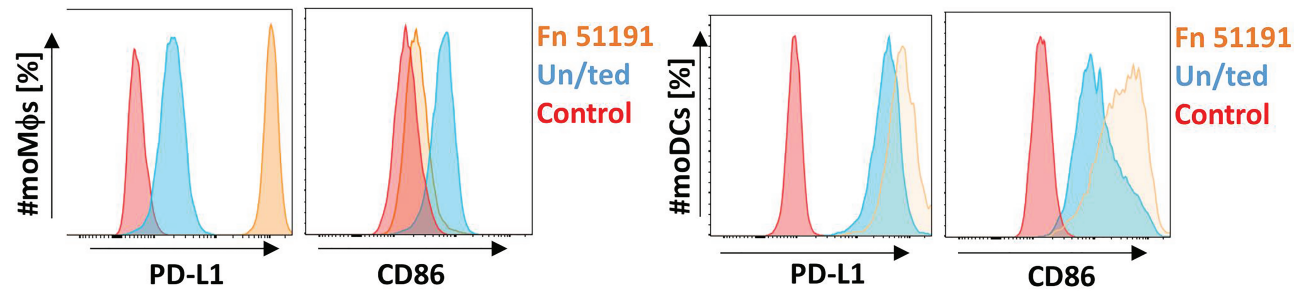

C
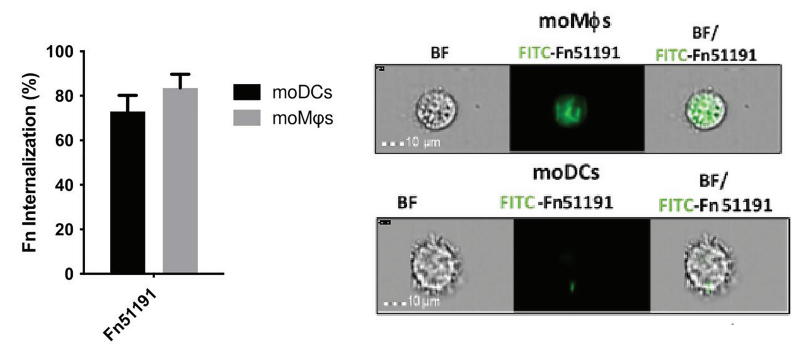

FIGURE 3 | Effect of F. nucleatum ATCC 51191 on human myeloid cells. Analysis of (A) cytokine and (B) cell surface marker expression in moDCs or moM $\phi s$ by flow cytometry. Human cells were stimulated with F. nucleatum ATCC 51191 (in orange). Unstained cells (in red) and unstimulated (un/ted) cells (in blue) were used as controls. (C) Internalisation of $F$. nucleatum ATCC 51191 into moDCs or moMps. Images were taken with a 40X objective. For the cytokine quantification, data shown are the mean of triplicates \pm SD derived from one representative experiment reproduced in three independent experiments. Statistical analyses were performed by one-way ANOVA followed by Tukey's test. $\mathrm{P}<0.05$ was considered as statistically significant. ${ }^{* \star \star *} \mathrm{p}<0.0001$, n.s., not statistically difference.

(Figure 4D). Stimulation of moM $\phi$ s with LPS (at 10 or $1 \mu \mathrm{g} / \mathrm{ml}$ ) showed a reduction of CD86 expression (Figure 4C), as also observed with the whole bacteria.

Overall, our results suggest that moDCs stimulated with F. nucleatum ATCC 51191 and derived components (OMVs and LPS) show a pro-inflammatory profile while F. nucleatumtreated moM $\phi$ s acquire a M2-phenotype which is associated with tumour progression (50).

\section{Siglec-7 Is Involved in F. nucleatum- Mediated Immune Response}

To obtain direct evidence of the contribution of Siglec-7 in F. nucleatum ATCC 51191 interaction with myeloid human cells, we used CRISPR-Cas9 editing to generate Siglec-7 deficient U-937 cells and assayed the effect of F. nucleatum stimulation on the immune response of differentiated U-937 WT or Siglec-7-deficient (Siglec-7/- ) cell lines (Figure 5). The expression of Siglec-7 in these cell lines was confirmed by flow cytometry (Figure S6). A significant increase $(\mathrm{p}<0.01)$ in TNF $\alpha$ and IL-10 cytokine production was observed in F. nucleatum ssp.-stimulated U-937-Siglec- $7^{-/-}$as compared to WT cells (Figure 5A).

Next, we carried out silencing of Siglec-7 in primary moDCs and moM $\phi$ s by siRNA. Using flow cytometry, we confirmed that Siglec-7 was expressed on the cell surface of moDCs and moM $\phi s$ (Figure S7A), and that expression could be reduced by up to $40 \%$ in moDCs as compared to the scramble control (Figure S7B) while no significant reduction in expression could be achieved in moM $\phi$ s. We then analysed the cytokine profile and expression of cell surface markers following stimulation of silenced or scramble control moDCs with F. nucleatum ATCC 51191 at MOI 5 (Figure 5B). We showed that stimulation of Siglec-7 silenced moDCs with $F$. nucleatum produced statistically significant $(\mathrm{p}<0.05)$ lower TNF $\alpha$ levels as compared to scramble moDCs (Figure 5B). No differences in cell surface marker expression were observed between F. nucleatumstimulated Siglec-7 silenced or scramble moDCs (Figure 5C). Together these data suggest that Siglec-7 is involved in the induction of a pro-inflammatory response in moDCs by $F$. nucleatum ATCC 51191. 
A

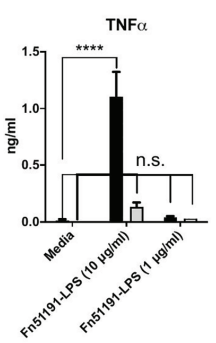

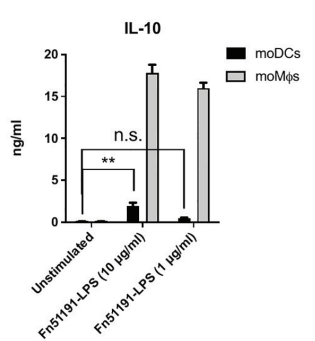

B
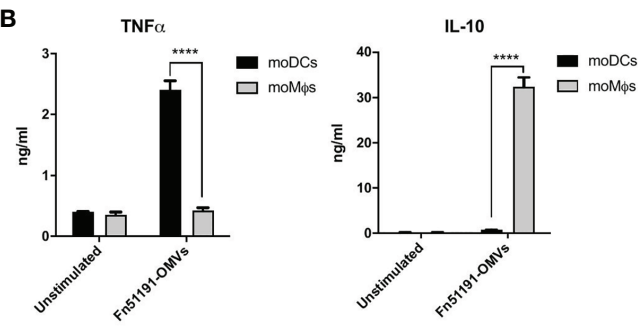

C
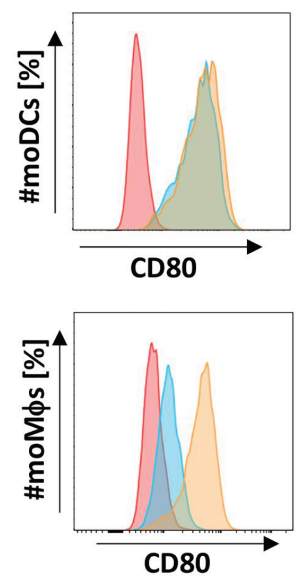

D
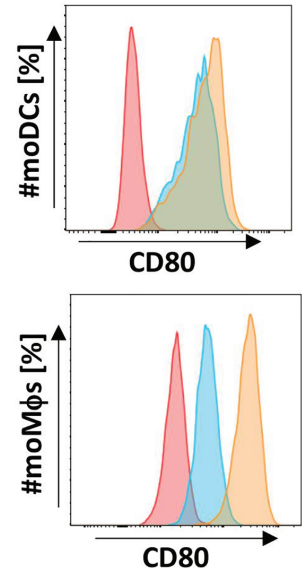

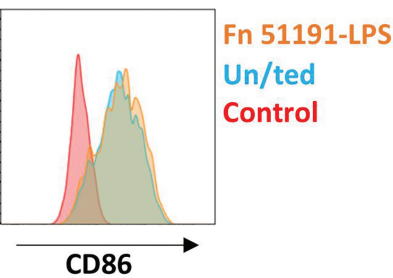

Fn 51191-LPS

Un/ted

Control
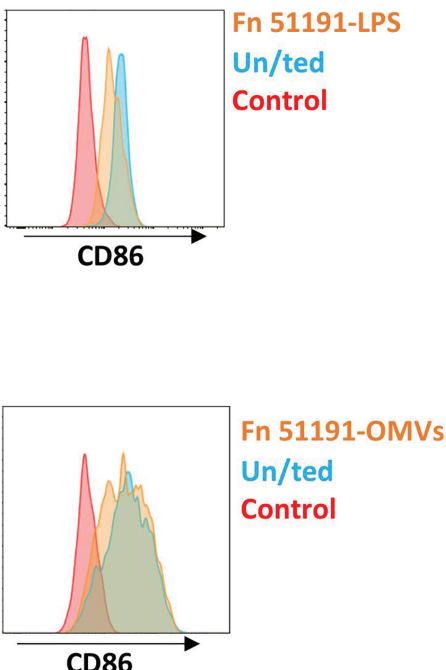

Fn 51191-OMVs

Un/ted

Control

FIGURE 4 | Effect of $F$. nucleatum ATCC 51191-derived LPS or OMVs on human myeloid cells. Analysis of (A) F. nucleatum LPS or (B) F. nucleatum OMVs on cytokine production and (C) F. nucleatum LPS or (D) F. nucleatum OMVs on cell surface marker expression. Unstimulated (un/ted) cells (in blue) and unstained cells (in red) were used as controls. Data shown are the mean of triplicates \pm SD derived from one representative experiment reproduced in three independent experiments. Statistical analyses were performed by one-way ANOVA followed by Tukey's test. $P<0.05$ was considered as statistically significant. ${ }^{* \star} p<0.01$, ${ }_{\star \star \star \star \star} p<0.0001$, n.s., not statistically difference.

\section{DISCUSSION}

F. nucleatum is the most abundant bacterial species in the colorectal tumour microenvironment with F. nucleatum ssp. animalis ATCC 51191 being enriched in CRC tissues (11). F. nucleatum encodes an array of genes related to adhesion and invasion (51), enabling it to reside intracellularly in tumour cells (52), and, once there, potentially influencing tumorigenesis. Adhesion and invasion of F. nucleatum to epithelial cells are mediated by the Fap2 lectin and FadA adhesin expressed on the surface of F. nucleatum. The Fap2 lectin interacts with GalGalNAc glycans which are overexpressed on tumour cells (53), 
A
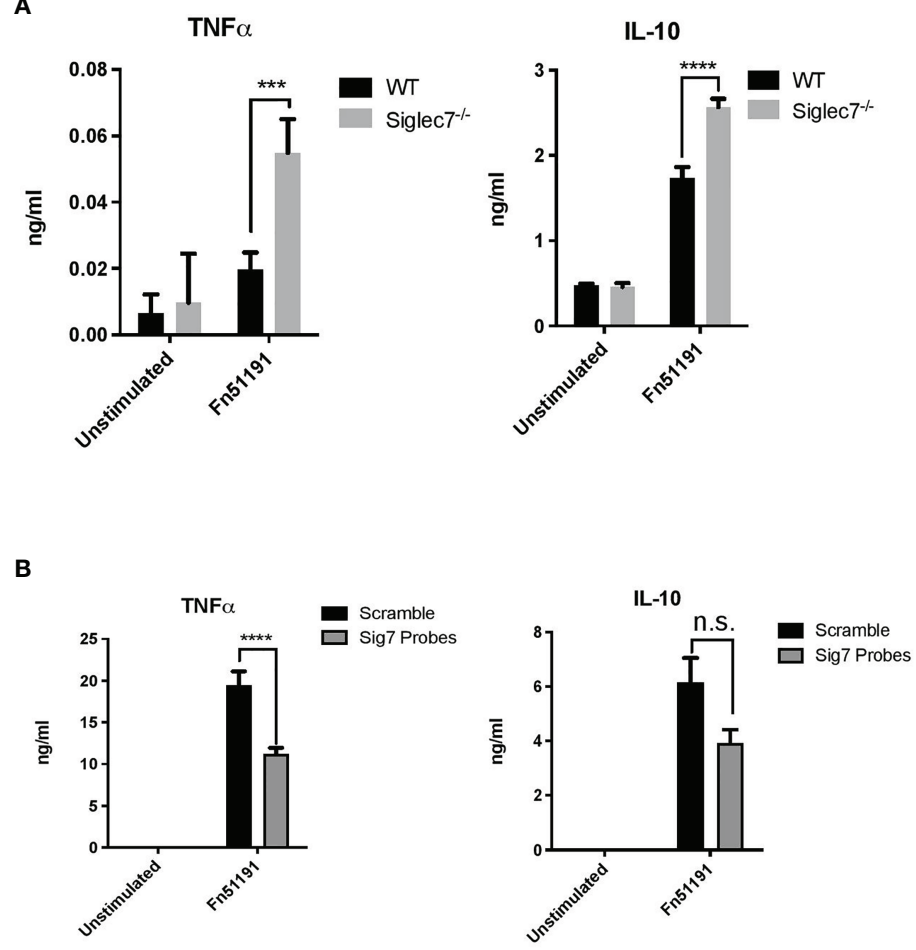

C

Fn ATCC 51191
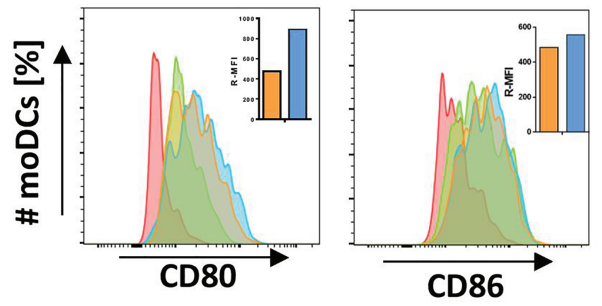

Control

Un/ted

Scramble + Fn strains

Siglec-7 probes + Fn

strains

FIGURE 5 | Effect of Siglec-7 on F. nucleatum ATCC 51191 interaction with human immune cells. (A) Cytokine production of U-937-PMA (WT or Siglec-7 ${ }^{-/}$) stimulated with F. nucleatum ATCC 51191. Bars represent the median values from 3 technical replicates. (B) Cytokine production and (C) cell surface marker expression of Siglec-7 silenced moDCs (in orange) or scramble control cells (in blue) stimulated with F. nucleatum. Unstimulated (un/ted) cells (in green) and unstained cells (in red) were used as controls. For the cytokine and internalisation analyses, data shown are the mean of triplicates \pm SD and duplicated \pm SD, respectively, derived from one representative experiment reproduced in three independent experiments. Statistical analyses were performed by two-way ANOVA followed by Tukey's test. $P<0.05$ was considered as statistically significant. ${ }^{\star \star \star} p<0.001,{ }^{\star \star \star \star} p<0.0001$, n.s., not statistically difference.

while the FadA adhesin, recognises and binds host surface components, such as vascular endothelial cadherin (54) and epithelial cadherin (16). F. nucleatum FadA expression was also shown to be upregulated in CRC tissue (16). Interaction of FadA with E-cadherin triggers the expression of oncogenes, such as $\mathrm{c}-\mathrm{MYC}$ and inflammatory genes, through the $\beta$-catenin cascade and the upregulation of annenix A1 $(16,17)$. However, the receptors involved in the interaction between F. nucleatum ssp. and immune cells remain largely unknown.

Here, we showed that F. nucleatum ssp. animalis ATCC 51191 interacts with Siglec-7 expressed by immune cells and that binding is LPS-mediated. Human NK cells, macrophages, dendritic cells constitutively express Siglec-7, and the colonic lamina propria monocytes and macrophages represent the major Siglec-7 positive populations (55). Extracellularly, Siglec-7 has a sialic acid-binding $\mathrm{V}$-set domain which we demonstrated was implicated in the binding to F. nucleatum. Siglec-7 has been shown to bind to the sialylated ganglioside GD3 (56), and Nlinked disialyl Lewis ${ }^{\mathrm{a}}$ in the normal colonic epithelium (55). At the molecular level, Siglec-7 has been reported to bind to terminal sialic acid moieties with diverse underlying glycan structures. We recently uncovered the LPS structure of $F$. nucleatum ATCC 51191, revealing a novel sugar repeating unit in the O-antigen structure $[\rightarrow 4)-\beta$-D-GlcpNAcA- $(1 \rightarrow 4)-\beta$-D- 
GlcpNAc3NAlaA-( $1 \rightarrow 3)-\alpha-D-F u c p N A c 4 N R-(1 \rightarrow], \quad(\mathrm{R}=$ Acetylated 60\%), and a bis-phosphorylated hexa-acylated lipid A moiety (24). It therefore likely that the LPS glycans other than sialic acid moieties may contribute to the binding of F. nucleatum ATCC 51191 to Siglec-7, consistent with the results of the sialidase treatment. This was further confirmed by STD NMR, showing that the OPS extracted from F. nucleatum ATCC 51191 was recognised by Siglec-7, revealing new ligand epitopes not restricted to nonulosonic acids (neuraminic acid and fusaminic acid). The discovery that $F$. nucleatum LPS is a ligand for immune checkpoint receptors like Siglec-7 opens up new blockade strategies and studies are in progress to gain further structural insights into the broad ligand specificity of Siglec-7 towards the bacterial glycan structures revealed in this work.

We showed that F. nucleatum ATCC 51191 induced a proinflammatory profile in moDCs and a tumour associated profile in macrophages (moM $\phi$ s and U-937 cells) and that Siglec-7 contributed to these cell-specific responses using Siglec-7 RNA-silenced moDCs and Siglec-7 deficient U-937 cells. In macrophages, F. nucleatum ATCC 51191 induced the expression of IL-10, IL-8 cytokines and PD-L1 marker and a downregulation of CD86 cell surface marker expression, characteristic of macrophage type 2 (M2) polarisation $(12,13)$. These results are in agreement with previous studies showing an infiltration of M2-macrophages in F. nucleatum ssp. positive clinical CRC specimens (57) and a M2 acquired phenotype in macrophage-like cell lines stimulated with F. nucleatum ATCC 10953 (13), and F. nucleatum ATCC 25586 (58). A recent study showed that Siglec-7 and -9 induce the polarisation of monocytes into a tumour-associated macrophage (TAM) phenotype and the induction of tumour-associated cell surface markers such as PD-L1 (59). The moDC response to F. nucleatum ATCC 51191 suggests that Siglec-7 contributes to the pro-inflammatory response. These cellspecific phenotypes could be recapitulated using $F$. nucleatum derived OMVs or LPS, implicating LPS as a ligand of the interaction with Siglec-7. The interaction of F. nucleatum derived LPS has been reported with TLR-4 leading to polarisation of macrophages, a process that is associated with tumour cell proliferation and metastasis (13). Our findings that LPS and OMVs influence innate immune cell responses is supported by recent studies showing that $F$. nucleatum OMVs can trigger inflammation of human intestinal epithelial cells (IECs) (60), by promoting NF- $\kappa B$ activation in a TLR-2-dependent manner (61).

Siglec-7 can bind to a range of human cell types (such as basophils, eosinophils, NK cells and splenocytes), illustrating its role of 'self recognition (62). The response we observed upon interaction with F. nucleatum differs from the canonical inhibition of immune activity observed between immune cells and cancer cells (25) but is consistent with in vivo work using ApcMin/+ model reporting that $F$. nucleatum induced expression of the genes encoding several pro-inflammatory cytokines, including TNF $\alpha$, IL-6, IL-8 and IL-1 $\beta$ (12) which mirrors human RNA-seq data from patients bearing high $F$. nucleatum loads in their colorectal tumours (12). To our knowledge, only one study, using Siglec-7 silencing approach in monocytes, also showed association of Siglec-7 with pro-inflammatory cytokine production, upon interaction with yeast particles in a sialic acid-independent manner (63). These differences in cell immune response could be attributed to the mode of recognition, the nature of the interactions (cell-cell or cellmicrobe), or the heterogeneity in pattern recognition receptor (PRR) expression in the different cell subsets used in the in vitro studies. Indeed, other PRRs may act synergistically with Siglec-7 to contribute to a distinct immune response. For example, TLR-4, a toll-like receptor with an intracellular activation motif has been shown to establish a direct interaction with Siglecs including Siglec7 (64). Therefore, since TLR-4 is expressed in U-937 (65) and moDCs (66), our findings could be the result of a synergetic effect between Siglec-7 and TLR-4. This interaction could also contribute to the capacity of $F$. nucleatum ssp. to promote chemoresistance of CRC by inhibition of cancer cell apoptosis (8-10). A recent in vivo study using humanized immunocompetent mice, showed that Siglec-7 and -9 could be potential targets to enhance anti-tumour immunity (36). In the future, it will be interesting to study the effect of F. nucleatum-Siglec-7 interaction in vivo, using humanised immunocompetent murine model, as Siglec-E, the closest murine homolog of Siglec-7, does not recognise F. nucleatum ssp. (data not shown), consistent with the lack of direct homology between murine and human Siglecs (67).

It was recently reported that Siglec-7 is expressed in macrophages in CRC tissue from patients and that high levels of Siglec-7 expression in tumour tissues are associated with shorter overall survival in patients treated with immunotherapy for metastatic CRC (68). Mirroring this, an independent human study reported that patients with high relative abundance of $F$. nucleatum in tumour tissues compared to matched control tissues have a higher incidence of regional lymph node metastases (69). These studies support the translation of our findings to humans. The interaction of F. nucleatum ssp. with Siglec-7, leading to a proinflammatory microenvironment, provides a mechanism underpinning these associations in patients and initial evidence that blocking this interaction may be a potential strategy to alleviate the progression of F. nucleatum associated CRC.

In summary, our results reporting LPS-mediated interaction of F. nucleatum and derived OMVs with Siglec-7, add a new dimension in our understanding of the role of Siglecs in cancer progression. Given the role of F. nucleatum in influencing CRC tumorigenesis and response to cancer treatment, there is significant interest in developing strategies that target $F$. nucleatum, preferably in the tumour tissue. However, antimicrobial strategies are limited due to concerns about antibiotic resistance and the mutualistic role of F. nucleatum in the oral cavity and other mucosal sites of humans (70). Targeted glycan interventions to displace Siglec-7-F. nucleatum interactions could prove an effective way of improving current approaches for the treatment of cancer by targeting $F$. nucleatum in the tumour environment and without compromising the rest of the gut microbiome or inducing antimicrobial resistance.

\section{DATA AVAILABILITY STATEMENT}

The original contributions presented in the study are included in the article/Supplementary Material. Further inquiries can be directed to the corresponding author. 


\section{ETHICS STATEMENT}

Blood collection in this study was approved by the Faculty of Medicine and Health Sciences Research Ethics Committee REC reference number 2013/2014 -14HT (University of East Anglia).

\section{AUTHOR CONTRIBUTIONS}

Conceptualization, DL and NJ. Experimental work, DL, PG-V, $\mathrm{KM}$, and RM. Resources and materials, NJ, PC, MM, CH, and KB. Writing - original draft preparation, NJ and DL. Review and editing, DL, PC, CC, and NJ. Supervision, NJ, AS, MM, CC, and KB. Funding acquisition, NJ. All authors contributed to the article and approved the submitted version.

\section{ACKNOWLEDGMENTS}

The authors gratefully acknowledge the support of the Biotechnology and Biological Sciences Research Council (BBSRC); this research was funded by the BBSRC Institute Strategic Programme Gut Microbes and Health BB/R012490/1

\section{REFERENCES}

1. The Global Cancer Observatory G. Source: Globocan 2018. World Heal Organ (2019) 876:2018-9.

2. Loomans-Kropp HA, Umar A. Increasing Incidence of Colorectal Cancer in Young Adults. J Cancer Epidemiol (2019) 2019:1-9. doi: 10.1155/2019/ 9841295

3. Keum NN, Giovannucci E. Global Burden of Colorectal Cancer: Emerging Trends, Risk Factors and Prevention Strategies. Nat Rev Gastroenterol Hepatol (2019) 16:713-32. doi: 10.1038/s41575-019-0189-8

4. Kostic AD, Gevers D, Pedamallu CS, Michaud M, Duke F, Earl AM, et al. Genomic Analysis Identifies Association of Fusobacterium With Colorectal Carcinoma. Genome Res (2012) 22:292-8. doi: 10.1101/gr.126573.111

5. Gao R, Kong C, Huang L, Li H, Qu X, Liu Z, et al. Mucosa-Associated Microbiota Signature in Colorectal Cancer. Eur J Clin Microbiol Infect Dis (2017) 36:2073-83. doi: 10.1007/s10096-017-3026-4

6. Dai Z, Coker OO, Nakatsu G, Wu WKK, Zhao L, Chen Z, et al. Multi-Cohort Analysis of Colorectal Cancer Metagenome Identified Altered Bacteria Across Populations and Universal Bacterial Markers. Microbiome (2018) 6:70. doi: 10.1186/s40168-018-0451-2

7. Kunzmann AT, Proença MA, Jordao HW, Jiraskova K, Schneiderova M, Levy M, et al. Fusobacterium Nucleatum Tumor DNA Levels are Associated With Survival in Colorectal Cancer Patients. Eur J Clin Microbiol Infect Dis (2019) 38:1891-9. doi: 10.1007/s10096-019-03649-1

8. Geller LT, Barzily-rokni M, Danino T, Jonas OH, Shental N, Nejman D, et al. Potential Role of Intratumor Bacteria in Mediating Tumor Resistance to the Chemotherapeutic Drug Gemcitabine. Science (2017) 1160:1156-60. doi: 10.1126/science.aah5043

9. Ramos A, Hemann MT. Drugs, Bugs, and Cancer: Fusobacterium Nucleatum Promotes Chemoresistance in Colorectal Cancer. Cell (2017) 170:411-3. doi: 10.1016/j.cell.2017.07.018

10. Yu TC, Guo F, Yu Y, Sun T, Ma D, Han J, et al. Fusobacterium Nucleatum Promotes Chemoresistance to Colorectal Cancer by Modulating Autophagy. Cell (2017) 170:548-63.e16. doi: 10.1016/j.cell.2017.07.008

11. Ye X, Wang R, Bhattacharya R, Boulbes D, Fan F, Xia L, et al. Fusobacterium Nucleatum Subspecies Animalis Influences Pro-Inflammatory Cytokine Expression and Monocyte Activation in Human Colorectal Tumors. Cancer Prev Res (2017) 10: canprevres.0178.2016. doi: 10.1158/1940-6207.CAPR-16-0178 and its constituent project BBS/E/F/000PR10353 (Theme 1, Determinants of microbe-host responses in the gut across life). DL was supported by the BBSRC Norwich Research Park Biosciences Doctoral Training Partnership grant number BB/ M011216/1. PGV acknowledges financial support from the European Commission via the International Training Network Train2Target (721484). AS was supported by PRIN 2017 (2017XZ2ZBK, 2019-2022), RM was supported by the European Research Council (ERC) under the European Union's Horizon 2020 research and innovation programme, grant agreement number 851356 . Work in the PRC lab was supported by Wellcome Trust grant 103744/Z/14/Z. MM acknowledges funding from GlycoNet, NSERC, and a Canada Tier II research chair in Chemical Glycoimmunology. KM acknowledges support through an Alberta Graduate Excellence Scholarship.

\section{SUPPLEMENTARY MATERIAL}

The Supplementary Material for this article can be found online at: https://www.frontiersin.org/articles/10.3389/fimmu.2021. 744184/full\#supplementary-material

12. Kostic AD, Chun E, Robertson L, Glickman JN, Gallini CA, Michaud M, et al Fusobacterium Nucleatum Potentiates Intestinal Tumorigenesis and Modulates the Tumor-Immune Microenvironment. Cell Host Microbe (2013) 14:207-15. doi: 10.1016/j.chom.2013.07.007

13. Chen T, Li Q, Wu J, Wu Y, Peng W, Li H, et al. Fusobacterium Nucleatum Promotes M2 Polarization of Macrophages in the Microenvironment of Colorectal Tumours via a TLR4-Dependent Mechanism. Cancer Immunol Immunother (2018) 67:1635-46. doi: 10.1007/s00262-018-2233-x

14. Mima K, Sukawa Y, Nishihara R, Qian ZR, Yamauchi M, Inamura K, et al. Fusobacterium Nucleatum and T Cells in Colorectal Carcinoma. JAMA Oncol (2015) 1:653-61. doi: 10.1001/jamaoncol.2015.1377

15. Yu X, Harden K, Gonzalez LC, Francesco M, Chiang E, Irving B, et al. The Surface Protein TIGIT Suppresses T Cell Activation by Promoting the Generation of Mature Immunoregulatory Dendritic Cells. Nat Immunol (2009) 10:48-57. doi: 10.1038/ni.1674

16. Rubinstein MR, Wang X, Liu W, Hao Y, Cai G, Han YW. Fusobacterium Nucleatum Promotes Colorectal Carcinogenesis by Modulating E-Cadherin/ $\beta$-Catenin Signaling via Its FadA Adhesin. Cell Host Microbe (2013) 14:195206. doi: 10.1016/j.chom.2013.07.012

17. Rubinstein MR, Baik JE, Lagana SM, Han RP, Raab WJ, Sahoo D, et al. Fusobacterium Nucleatum Promotes Colorectal Cancer by Inducing Wnt/ $\beta$ Catenin Modulator Annexin A1. EMBO Rep (2019) 8:e47638. doi: 10.15252/ embr.201847638

18. Yan X, Liu L, Li H, Qin H, Sun Z. Clinical Significance of Fusobacterium Nucleatum, Epithelial-Mesenchymal Transition, and Cancer Stem Cell Markers in Stage III/IV Colorectal Cancer Patients. Onco Targets Ther (2017) 10:5031-46. doi: 10.2147/OTT.S145949

19. Vinogradov E, St Michael F, Cox AD. Structure of the LPS O-Chain From Fusobacterium Nucleatum Strain ATCC 23726 Containing a Novel 5,7Diamino-3,5,7,9-Tetradeoxy-L-Gluco-non-2-Ulosonic Acid Presumably Having the D-Glycero-L-Gluco Configuration. Carbohydr Res (2018) 468:69-72. doi: 10.1016/j.carres.2018.08.011

20. Vinogradov E, Michael FS, Cox AD. The Structure of the LPS O-Chain of Fusobacterium Nucleatum Strain 25586 Containing Two Novel Monosaccharides, 2-Acetamido-2 , 6- non-2-Ulosonic Acid. Carbohydr Res (2017) 440-441:10-5. doi: 10.1016/j.carres.2017.01.002

21. Vinogradov E, St. Michael F, Homma K, Sharma A, Cox AD. Structure of the LPS O-Chain From Fusobacterium Nucleatum Strain 10953, Containing Sialic 
Acid. Carbohydr Res (2017) 440-441:38-42. doi: 10.1016/J.CARRES. 2017.01.009

22. Vinogradov E, St. Michael F, Cox AD. Structure of the LPS O-Chain From Fusobacterium Nucleatum Strain 12230. Carbohydr Res (2017) 448:115-7. doi: 10.1016/j.carres.2017.06.007

23. Vinogradov E, St Michael F, Cox AD. Structure of the LPS O-Chain From Fusobacterium Nucleatum Strain MJR 7757 B. Carbohydr Res (2018) 463:379. doi: 10.1016/j.carres.2018.04.010

24. Garcia-Vello P, Di Lorenzo F, Lamprinaki D, Notaro A, Speciale I, Molinaro A, et al. Structure of the O-Antigen and the Lipid A From the Lipopolysaccharide of Fusobacterium Nucleatum ATCC 51191. ChemBioChem (2020) 22:cbic.202000751. doi: 10.1002/cbic.202000751

25. Brown GD, Crocker PR. Lectin Receptors Expressed on Myeloid Cells. Microbiol Spectr (2016) 4:1-26. doi: 10.1128/microbiolspec.mchd-0036-2016

26. Crocker PR, Paulson JC, Varki A. Siglecs and Their Roles in the Immune System. Nat Rev Immunol (2007) 7:255-66. doi: 10.1038/nri2056

27. Van de Wall S, Santegoets KCM, van Houtum EJH, Büll C, Adema GJ. Sialoglycans and Siglecs Can Shape the Tumor Immune Microenvironment. Trends Immunol (2020) 41:274-85. doi: 10.1016/j.it.2020.02.001

28. Macauley MS, Crocker PR, Paulson JC. Siglec-Mediated Regulation of Immune Cell Function in Disease. Nat Rev Immunol (2014) 14:653-66. doi: $10.1038 /$ nri3737

29. Varki A, Gagneux P. Multifarious Roles of Sialic Acids in Immunity. Ann N Y Acad Sci (2012) 1253:16-36. doi: 10.1111/j.1749-6632.2012.06517.x

30. Jandus C, Simon HU, Von Gunten S. Targeting Siglecs-A Novel Pharmacological Strategy for Immuno- and Glycotherapy. Biochem Pharmacol (2011) 82:323-32. doi: 10.1016/j.bcp.2011.05.018

31. Manni M, Läubli H. Targeting Glyco-Immune Checkpoints for Cancer Therapy. Expert Opin Biol Ther (2021) 21(8):1063-71. doi: 10.1080/14712598. 2021.1882989

32. Smith BAH, Bertozzi CR. The Clinical Impact of Glycobiology: Targeting Selectins, Siglecs and Mammalian Glycans. Nat Rev Drug Discovery (2021) 20:217-43. doi: 10.1038/s41573-020-00093-1

33. Wisnovsky S, Möckl L, Malaker SA, Pedram K, Hess GT, Riley NM, et al. Genome-Wide CRISPR Screens Reveal a Specific Ligand for the GlycanBinding Immune Checkpoint Receptor Siglec-7. Proc Natl Acad Sci USA (2021) 118(5):e2015024118. doi: 10.1073/pnas.2015024118

34. Xiao H, Woods EC, Vukojicic P, Bertozzi CR. Precision Glycocalyx Editing as a Strategy for Cancer Immunotherapy. Proc Natl Acad Sci USA (2016) 113:10304-9. doi: 10.1073/pnas.1608069113

35. Fraschilla I, Pillai S. Viewing Siglecs Through the Lens of Tumor Immunology. Immunol Rev (2017) 276:178-91. doi: 10.1111/imr.12526

36. Ibarlucea-Benitez I, Weitzenfeld P, Smith P, Ravetch JV. Siglecs-7/9 Function as Inhibitory Immune Checkpoints In Vivo and can be Targeted to Enhance Therapeutic Antitumor Immunity. Proc Natl Acad Sci (2021) 118: e2107424118. doi: 10.1073/pnas.2107424118

37. Heikema AP, Bergman MP, Richards H, Crocker PR, Gilbert M, Samsom JN, et al. Characterization of the Specific Interaction Between Sialoadhesin and Sialylated Campylobacter Jejuni Lipooligosaccharides. Infect Immun (2010) 78:3237-46. doi: 10.1128/IAI.01273-09

38. Stephenson HN, Mills DC, Jones H, Milioris E, Copland A, Dorrell N, et al. Pseudaminic Acid on Campylobacter Jejuni Flagella Modulates Dendritic Cell IL-10 Expression via Siglec-10 Receptor: A Novel Flagellin-Host Interaction. J Infect Dis (2014) 210:1487-98. doi: 10.1093/infdis/jiu287

39. Fong JJ, Tsai C-M, Saha S, Nizet V, Varki A, Bui JD. Siglec-7 Engagement by GBS $\beta$-Protein Suppresses Pyroptotic Cell Death of Natural Killer Cells. Proc Natl Acad Sci (2018) 115:10410-5. doi: 10.1073/pnas.1804108115

40. Angata T. Possible Influences of Endogenous and Exogenous Ligands on the Evolution of Human Siglecs. Front Immunol (2018) 9:2885. doi: 10.3389/ fimmu.2018.02885

41. De Castro C, Parrilli M, Holst O, Molinaro A. Microbe-Associated Molecular Patterns in Innate Immunity. In: Methods in Enzymology. Academic Press (2010). p. 89-115. doi: 10.1016/S0076-6879(10)80005-9

42. Tsai C-M, Frasch CE. A Sensitive Silver Stain for Detecting Lipopolysaccharides in Polyacrylamide Gels. Anal Biochem (1982) 119:115-9. doi: 10.1016/0003-2697 (82) $90673-\mathrm{X}$

43. Liu J, Hsieh CL, Gelincik O, Devolder B, Sei S, Zhang S, et al. Proteomic Characterization of Outer Membrane Vesicles From Gut Mucosa-Derived
Fusobacterium Nucleatum. J Proteomics (2019) 195:125-37. doi: 10.1016/ j.jprot.2018.12.029

44. Hase S, Hofstad T, Rietschel ET. Chemical Structure of the Lipid A Component of Lipopolysaccharides From Fusobacterium Nucleatum. J Bacteriol (1977) 129:9-14. doi: 10.1128/JB.129.1.9-14.1977

45. Ohradanova-Repic A, Machacek C, Fischer MB, Stockinger H. Differentiation of Human Monocytes and Derived Subsets of Macrophages and Dendritic Cells by the HLDA10 Monoclonal Antibody Panel. Clin Transl Immunol (2016) 5:e55-9. doi: 10.1038/cti.2015.39

46. Sintiprungrat K, Singhto N, Sinchaikul S, Chen ST, Thongboonkerd V. Alterations in Cellular Proteome and Secretome Upon Differentiation From Monocyte to Macrophage by Treatment With Phorbol Myristate Acetate: Insights Into Biological Processes. J Proteomics (2010) 73:602-18. doi: 10.1016/ j.jprot.2009.08.001

47. Troegeler A, Lastrucci C, Duval C, Tanne A, Cougoule C, MaridonneauParini I, et al. An Efficient siRNA-Mediated Gene Silencing in Primary Human Monocytes, Dendritic Cells and Macrophages. Immunol Cell Biol (2014) 92:699-708. doi: 10.1038/icb.2014.39

48. Hashimoto N, Ito S, Tsuchida A, Bhuiyan RH, Okajima T, Yamamoto A, et al. The Ceramide Moiety of Disialoganglioside (GD3) Is Essential for GD3 Recognition by the Sialic Acid- Binding Lectin SIGLEC7 on the Cell Surface. J Biol Chem (2019) 294:10833-45. doi: 10.1074/jbc.RA118.007083

49. Marchetti R, Perez S, Arda A, Imberty A, Jimenez-Barbero J, Silipo A, et al. "Rules of Engagement" of Protein-Glycoconjugate Interactions: A Molecular View Achievable by Using NMR Spectroscopy and Molecular Modeling. ChemistryOpen (2016) 5:274-96. doi: 10.1002/open.201600024

50. Sica A, Schioppa T, Mantovani A, Allavena P. Tumour-Associated Macrophages Are a Distinct M2 Polarised Population Promoting Tumour Progression: Potential Targets of Anti-Cancer Therapy. Eur J Cancer (2006) 42:717-27. doi: 10.1016/j.ejca.2006.01.003

51. Mcguire AM, Cochrane K, Griggs AD, Mcguire AM, Cochrane K, Griggs AD, et al. Evolution of Invasion in a Diverse Set of Fusobacterium Species. MBio (2014) 5:1-11. doi: 10.1128/mBio.01864-14.Editor

52. Yamamura K, Izumi D, Kandimalla R, Sonohara F, Baba Y, Yoshida N, et al. Intratumoral Fusobacterium Nucleatum Levels Predict Therapeutic Response to Neoadjuvant Chemotherapy in Esophageal Squamous Cell Carcinoma. Clin Cancer Res (2019) 25:6170-9. doi: 10.1158/1078-0432. CCR-19-0318

53. Gur C, Ibrahim Y, Isaacson B, Yamin R, Abed J, Gamliel M, et al. Binding of the Fap2 Protein of Fusobacterium Nucleatum to Human Inhibitory Receptor TIGIT Protects Tumors From Immune Cell Attack. Immunity (2015) 42:34455. doi: 10.1016/j.immuni.2015.01.010

54. Fardini Y, Wang X, Témoin S, Nithianantham S, Lee D, Shoham M, et al. Fusobacterium Nucleatum Adhesin FadA Binds Vascular Endothelial Cadherin and Alters Endothelial Integrity. Mol Microbiol (2011) 82:146880. doi: $10.1111 / j .1365-2958.2011 .07905 . x$

55. Miyazaki K, Sakuma K, Kawamura YI, Izawa M, Ohmori K, Mitsuki M, et al. Colonic Epithelial Cells Express Specific Ligands for Mucosal Macrophage Immunosuppressive Receptors Siglec-7 and -9. J Immunol (2012) 188:4690700. doi: 10.4049/jimmunol.1100605

56. Nicoll G, Avril T, Lock K, Furukawa K, Bovin N, Crocker PR. Ganglioside GD3 Expression on Target Cells can Modulate NK Cell Cytotoxicity via Siglec-7-Dependent and -Independent Mechanisms. Eur J Immunol (2003) 33:1642-8. doi: 10.1002/eji.200323693

57. Park HE, Kim JH, Cho NY, Lee HS, Kang GH. Intratumoral Fusobacterium Nucleatum Abundance Correlates With Macrophage Infiltration and CDKN2A Methylation in Microsatellite-Unstable Colorectal Carcinoma. Virchows Arch (2017) 471:329-36. doi: 10.1007/s00428-017-2171-6

58. Hu L, Liu Y, Kong X, Wu R, Peng Q, Zhang Y, et al. Fusobacterium Nucleatum Facilitates M2 Macrophage Polarization and Colorectal Carcinoma Progression by Activating TLR4/NF-kb/S100A9 Cascade. Front Immunol (2021) 12:658681. doi: 10.3389/fimmu.2021.658681

59. Rodriguez E, Boelaars K, Brown K, Eveline Li RJ, Kruijssen L, Bruijns SCM, et al. Sialic Acids in Pancreatic Cancer Cells Drive Tumour-Associated Macrophage Differentiation via the Siglec Receptors Siglec-7 and Siglec-9. Nat Commun (2021) 12:1-14. doi: 10.1038/s41467-021-21550-4

60. Engevik MA, Danhof HA, Ruan W, Engevik AC, Chang-Graham AL, Engevik KA, et al. Fusobacterium Nucleatum Secretes Outer Membrane Vesicles and 
Promotes Intestinal Inflammation. MBio (2021) 12:1-17. doi: 10.1128/ mBio.02706-20

61. Martin-Gallausiaux C, Malabirade A, Habier J, Wilmes P. Fusobacterium Nucleatum Extracellular Vesicles Modulate Gut Epithelial Cell Innate Immunity via FomA and TLR2. Front Immunol (2020) 11:583644. doi: 10.3389/fimmu.2020.583644

62. Rodrigues E, Jung J, Park H, Loo C, Soukhtehzari S, Kitova EN, et al. A Versatile Soluble Siglec Scaffold for Sensitive and Quantitative Detection of Glycan Ligands. Nat Commun (2020) 11:5091. doi: 10.1038/s41467-02018907-6

63. Varchetta S, Brunetta E, Roberto A, Mikulak J, Hudspeth KL, Mondelli MU, et al. Engagement of Siglec-7 Receptor Induces a Pro-Inflammatory Response Selectively in Monocytes. PloS One (2012) 7(9):e45821. doi: 10.1371/ journal.pone.0045821

64. Chen G-Y, Brown NK, Wu W, Khedri Z, Yu H, Chen X, et al. Broad and Direct Interaction Between TLR and Siglec Families of Pattern Recognition Receptors and its Regulation by Neu1. Elife (2014) 3:1-18. doi: 10.7554/elife.04066

65. Greene CM, McElvaney NG, O’Neill SJ, Taggart CC. Secretory Leucoprotease Inhibitor Impairs Toll-Like Receptor 2- and 4-Mediated Responses in Monocytic Cells. Infect Immun (2004) 72:3684-7. doi: 10.1128/IAI.72.6.3684-3687.2004

66. Novak N, Koch S, Allam J-P, Bieber T. Dendritic Cells: Bridging Innate and Adaptive Immunity in Atopic Dermatitis. J Allergy Clin Immunol (2010) 125:50-9. doi: 10.1016/j.jaci.2009.11.019

67. Yu Z, Maoui M, Wu L, Banville D, Shen S. Msiglec-E, a Novel Mouse CD33Related Siglec (Sialic Acid-Binding Immunoglobulin-Like Lectin) That Recruits Src Homology 2 (SH2)-Domain-Containing Protein Tyrosine Phosphatases SHP-1 and SHP-2. Biochem J (2001) 353:483-92. doi: 10.1042/0264-6021:3530483
68. Yamada K, Hazama S, Suzuki N, Xu M, Nakagami Y, Fujiwara N, et al. Siglec7 Is a Predictive Biomarker for the Efficacy of Cancer Vaccination Against Metastatic Colorectal Cancer. Oncol Lett (2021) 21:1-11. doi: 10.3892/ ol.2020.12271

69. Castellarin M, Warren RL, Freeman JD, Warren L, Freeman JD, Dreolini L, et al. Fusobacterium Nucleatum Infection is Prevalent in Human Colorectal Carcinoma. Genome Res (2011) 22:299-306. doi: 10.1101/gr.126516.111

70. Brennan CA, Garrett WS. Fusobacterium Nucleatum - Symbiont, Opportunist and Oncobacterium. Nat Rev Microbiol (2019) 17:156-66. doi: 10.1038/s41579-018-0129-6

Conflict of Interest: The authors declare that the research was conducted in the absence of any commercial or financial relationships that could be construed as a potential conflict of interest.

Publisher's Note: All claims expressed in this article are solely those of the authors and do not necessarily represent those of their affiliated organizations, or those of the publisher, the editors and the reviewers. Any product that may be evaluated in this article, or claim that may be made by its manufacturer, is not guaranteed or endorsed by the publisher.

Copyright (C) 2021 Lamprinaki, Garcia-Vello, Marchetti, Hellmich, McCord, Bowles, Macauley, Silipo, De Castro, Crocker and Juge. This is an open-access article distributed under the terms of the Creative Commons Attribution License (CC BY). The use, distribution or reproduction in other forums is permitted, provided the original author(s) and the copyright owner(s) are credited and that the original publication in this journal is cited, in accordance with accepted academic practice. No use, distribution or reproduction is permitted which does not comply with these terms. 